\title{
Assessment of Muscle Function in Severe and Malnourished COVID-19 Patients
}

\author{
Marine Gerard ${ }^{1}$, Phi-Linh Nguyen-Thi ${ }^{2}$, Aurelie Malgras ${ }^{1}$, Thomas Bermand ${ }^{3}$, Roland Jaussaud $^{3}$, \\ Didier Quilliot ${ }^{1, *}$ \\ ${ }^{1}$ Transversal Unit of Nutrition, Department of Endocrinology, Diabetology and Nutrition, University of Lorraine, CHRU Nancy, Brabois \\ Hospital, Nancy, France \\ ${ }^{2}$ Medical Evaluation Department, Department of Clinical Research Support PARC, University of Lorraine, CHU Nancy, France \\ ${ }^{3}$ Internal Medicine and Clinical Immunology Department, Nancy University Hospital, Nancy, France
}

Email address:

d.quilliot@chru-nancy.fr (D. Quilliot)

${ }^{*}$ Corresponding author

\section{To cite this article:}

Marine Gerard, Phi-Linh Nguyen-Thi, Aurelie Malgras, Thomas Bermand, Roland Jaussaud, Didier Quilliot. Assessment of Muscle Function in Severe and Malnourished COVID-19 Patients. International Journal of Nutrition and Food Sciences. Vol. 9, No. 5, 2020 , pp. $132-137$. doi: $10.11648 /$ j.ijnfs.20200905.12

Received: November 3, 2020; Accepted: November 13, 2020; Published: November 23, 2020

\begin{abstract}
COVID-19 is a disease at high risk of muscle failure and undernutrition. In this setting, systematic screenings are necessary to assess muscle deficit during hospitalization and after discharge. Objective: To analyze the interest of a selfassessment of muscle strength (SES) to evaluate the evolution of muscle strength during COVID-19 and to assess the agreement between SES and the handgrip test. Methods: Prospective cohort study including all inpatients diagnosed with COVID-19 admitted in a non-ICU unit, until the required number of subjects is reached. Handgrip test and SES were recorded at admission and every two days during hospitalization and at Day30 post-discharge. Sarcopenic screening test (SARC-F) and International Physical Activity Questionnaire (IPAQ-SF) were administered before admission and on Day30. Nutritional status was recorded at admission, at discharge and at Day30 post-discharge. Evolution effects were analyzed using ANOVA for repeated measures and Pearson's chi-square test $(\mathrm{p}<0.05)$. Results: Handgrip and SES progression were significantly correlated $(\mathrm{p}=0.004)$, SES enabled detecting $95 \%$ of patients with progression in muscle strength. Muscle failure incidence was lower at discharge ( $73 \%$ to $42 \%$ for handgrip and $69 \%$ to $42 \%$ for SES ( $\mathrm{p}=0.0035)$ ). At admission, $61 \%$ had $\geq 30 \%$ reduction in food intake which was higher in the presence of anosmia and inflammation; $73 \%$ presented malnutrition and $19 \%$ necessitated tube feeding due to severe malnutrition as well as insufficient nutrition intake. Weight loss kinetics decreased significantly during hospitalization $(-0.4 \pm 1.6 \%$ vs. $5.4 \pm 6.3 \%$ pre-admission, $\mathrm{p}=0.0016$ ). At Day $30,17 \%$ had a severe muscle failure (SES $<7$ and/or SARC-F $>6$ ) and $32 \%$ had persistent severe malnutrition. Conclusion: The present study showed that three quarters of COVID-19 patients admitted in a nonICU setting presented malnutrition as well as sarcopenia as assessed by hand strength. Screening for malnutrition and muscle failure should be initiated immediately at the onset of care, with the aim of improving nutritional status as well as maintaining muscle mass and physical performance. During hospitalization, grip strength measured by a handheld dynamometer is inexpensive and easy to administer, even in a COVID unit. On the other hand, the 10-point verbal or visual analogue scales (SES) could prove useful in assessing the long-term progression of muscle strength.
\end{abstract}

Keywords: COVID-19, Sarcopenia, Muscle Failure, Malnutrition, Handgrip, Self-Evaluation

\section{Clinically Relevancy Statement}

COVID-19 is a disease at high risk muscle failure and undernutrition and a situation where muscle mass cannot be readily assessed. Muscle strength, e.g. handgrip strength, is an appropriate supporting proxy under the cover of systematic decontamination, albeit time-consuming for healthcare teams. However, a simpler method could be useful to assess the evolution of muscle deficit. This study was aimed at evaluating the interest of 10-point verbal or visual analogue scales to 
assess their muscle strength improvement and estimating the incidence of muscle failure and their respective evolution in a cohort of non-ICU COVID-19 patients at admission, during hospitalization, and to assess the evolution of nutrition and muscle status at 30 days after discharge. Our observations show that these patients had a good perception of their muscle strength improvement and that 10-point verbal or visual analogue scales (SES) could prove useful to assess the progression of muscle strength and ultimately be used to screen/follow muscle failure evolution of outpatients. As expected, our results showed that, at admission, three-quarters presented severe muscle failure as assessed by hand strength while two-thirds of the patients exhibited malnutrition.

\section{Introduction}

As with many acute infections, COVID-19 is a disease at high risk of undernutrition [1] that can potentially mask protein malnutrition (sarcopenia). The probability that the infected patient is already malnourished on admission to hospital is therefore high. Moreover, inactivity and bed rest accelerate muscle catabolism. Olfactory and gustatory dysfunction are common symptoms in patients with COVID19 [2] and may contribute to weight loss in other diseases, thereby increasing the risk of malnutrition [3]. Malnutrition can be prevented or contained by prompt nutritional support [6]. Early nutritional supplementation of non-ICU patients hospitalized for COVID-19 has been recommended by the French-speaking Society for Clinical Nutrition and Metabolism [7] as well as European Society for Clinical Nutrition and Metabolism (ESPEN) experts [8].

While loss of muscle quantity or function is particularly useful, the measurement of body composition remains difficult however in cases of infectious disease. Accordingly, COVID is a situation where muscle mass cannot be readily assessed. Grip strength measurement comprises standardized protocols of measurement and features available robust and validated cut-off values, which is not the case for all lower limb muscle strength values. The common use of handgrip strength in daily clinical practice has recently been highlighted in a survey based on clinicians experience [4] and has been shown to be a surrogate for lower extremity muscle strength due to its ease of measurement [5]. The use of grip strength is furthermore typically recommended as a supportive measure in the GLIM consensus [6]. During hospitalization for COVID-19, muscle strength, e.g. handgrip strength, is hence an appropriate supporting proxy under the cover of systematic decontamination, albeit time-consuming for healthcare teams. However, this test is not easily usable to track the progress of muscle strength at home or in post-care facilities. The sarcopenic screening test (Sarc-F) is a validated screening tool for remote consultation in primary care, although conversely not adapted to hospitalized or dyspneic patients [7]. A simpler method is thus necessary to assess the evolution of muscle deficit such as the evaluation of food intake by the Self Evaluation of Food Intake (SEFI) using a visual analogic scale for diagnostic purposes, as well as to guide nutritional treatment [8].

In light of the above, this study was to analyze the interest of a self-assessment of muscle strength (SES) to evaluate the evolution of muscle strength during COVID-19 and to assess the agreement between SES and the handgrip test. Secondary objectives included estimating the incidence of malnutrition and muscle failure and their respective evolution in a cohort of non-ICU COVID-19 patients at admission, during hospitalization as well as to assess the evolution of nutrition and muscle status at 30 days after discharge.

\section{Methods}

\subsection{Study Design and Participants}

This study included all adult inpatients ( $\geq 18$ years old) who were diagnosed with COVID-19 admitted in a non-ICU unit for COVID-19 patients at the Nancy Brabois University Hospital until the required number of subjects is reached. Patients who no longer justified hospitalization in intensive care but still required care in a non-ICU care unit were also included. ClinicalTrials.gov Identifier is NCT04451694.

Epidemiological, demographic, clinical, laboratory, treatment and outcome data were extracted from electronic medical records. The confirmation of SARS-CoV-2 infection was achieved by real-time RT-PCR methods.

\subsection{Nutritional Assessment}

Nutritional status was assessed using anthropometric measurements (BMI: body mass index=body weight $/$ height $^{2}$ ), weight loss within 1 month, and an index of food intake using the 10-point verbal (AVeS) or visual (AViS) analogue scales (SEFI or self-evaluation of food intake) [9]. Nutritional risk screening (NRS-2002) was systematically calculated [10] and diagnosis of malnutrition was made according to the GLIM criteria (at least 1 phenotypic criterion and 1 etiological criterion) [6]. As suggested by the French-speaking Society for Clinical Nutrition and Metabolism, the severity of malnutrition was defined as a BMI $<17$, weight loss $>10 \%$ within 1 month or if albuminemia was above $30 \mathrm{~g} / \mathrm{L}$ [11]. These parameters were evaluated both at admission and prior to discharge.

\subsection{Assessment of Muscle Function}

The Handgrip test and Self Evaluation of Strength (SES) were performed every 2 days during hospitalization to follow the progress of these 2 tests in parallel. The handgrip test (Jamar ${ }^{\circledR}$ ), was performed according to standard procedure [12]. An average of three trials with the dominant hand was collected. In accordance with the European consensus on the definition and diagnosis of sarcopenia [13], sarcopenia was suspected below a mean value $<16 \mathrm{~kg}$ for women and $26 \mathrm{~kg}$ for men. Patients were asked to evaluate their arm strength in comparison with their strength prior to COVID-19 and was performed every 2 days. As suggested by Krznaric et al.[14], patients were asked their degree of difficulty in lifting or carrying a weight, walking across the room, rising from a chair or bed, and to evaluate these 
difficulties using a 10-point verbal or visual analogue scale (selfevaluation of strength, SES). SARC-F [14] was also assessed at admission and at Day 30.

Assessment of physical activity Physical activities of the patients were queried by completing the International Physical Activity Questionnaire-Short Form (IPAQ-SF) in order to estimate activity prior to COVID-19: activities were classified as low physical activity $(<600)$ metabolic equivalents $[\mathrm{MET}] \times$ minutes per week or $<150$ minutes per week of moderate-intensity physical activity), moderate physical activity (600-3000 MET $\times$ minutes or $150-750$ minutes per week) and high physical activity $(>3000$ MET $\times$ minutes or $>750$ minutes per week) [15]. This evaluation was repeated at D30 after discharge.

\subsection{Nutritional Counseling and Nutritional Support}

As proposed by ESPEN [16], oral nutrition support included regular food and fortified food (meals or snacks), and oral nutritional supplements (ONS) were systematically prescribed for all patients on the first day of admission. The effect of systematically-administered dietary intervention was assessed every 2 days, with an evaluation of food intake (reduced food intake less than 30\%). When necessary, these patients underwent refeeding syndrome prevention with an intravenous supplementation of multivitamin, multimineral and trace element solutions, phosphorus and potassium.

In the case of inefficacity of dietary intervention (both nutritional counseling and oral supplements), a weight loss $>$ $10 \%$, or albuminemia $<30 \mathrm{~g} / \mathrm{L}$, or if $\mathrm{BMI}<18.5(<21$ if age $>70$ years) [9] and no increase in food intake in the 3-4 days after admission, enteral tube feeding (ETF) was systematically offered. When administered, ETF was progressively implemented with increments up to $80-100 \%$ of energy requirement after DAY 3 with $1.3 \mathrm{~g} / \mathrm{kg}$ "adjusted body weight" protein equivalents per day.

\subsection{Day 30 Evaluation}

Remote or phone consultation was conducted 30 days after discharge to evaluate weight, SEFI, anosmia, SARC-F and Self Evaluation of Strength, and Physical Activity (IPAQ-SF).

The study was approved by the Research Commission of University Hospital of Nancy and the requirement for informed consent was waived by the ethics commission.

\subsection{Statistical Analysis}

Sample size determination was based on calculation of number of pairs (repeated measurements) of handgrip evolution expected 23\%; \% normal handgrip reference estimated first measurement: $60 \%$; correlation between the 2 measurements $=0.5$, power $=80 \%$, alpha $=0.05$. Number of patient pairs included $=28$

Results are expressed as percentages, means \pm standard deviation. Evolution effects were analyzed using ANOVA for repeated measures and Pearson's chi-square test. Variables were deemed significant at the 0.05 level. Data were recorded on Excel ${ }^{\circledR}$ files. All analyses were performed using JMP trial 15.1.0 (SAS institute inc. 2019.

\section{Results}

Thirty patients hospitalized in the non-ICU COVID-19 unit were screened: 3 patients died during hospitalization and 1 was re-oriented to the ICU for pneumonia exacerbation and 26 were included. Mean age of the patients was $68.2 \pm 15.1$ years, $80.8 \%$ of whom were male. As described in Table 1 , $70 \%$ of these patients were overweight or obese and the majority had a high or moderate physical activity assessed by IPAQ-SF, prior to contracting COVID-19 (64.4\%). Anosmia was present in $8 / 26$ patients $(30 \%)$. The most frequent comorbidities were cardiovascular diseases (hypertension and coronary artery disease). Fourteen of the 23 patients were initially admitted in ICU (median period of 3 days (range 040). Mean duration of hospitalization was $9.8 \pm 4.8$ days (median=8, range 2-32).

\subsection{Assessment of Muscle Function (Table 2)}

At admission, handgrip strength was below $16 \mathrm{~kg}$ (for women) or $26 \mathrm{~kg}$ (for men) in 19/26 patients (73\%). Eight patients had a mid-arm circumference below $24 \mathrm{~cm}(\mathrm{~W})$ or $26 \mathrm{~cm}(\mathrm{M}) ; 21 / 26$ had a SARC-F $>4$.

During hospitalization, handgrip values increased significantly, and the number of patients with sarcopenia decreased from $19 / 26(73 \%)$ to $11 / 26(42 \%)$.

The increase in SES (\%) was significantly correlated with the progression of handgrip values $(\%)(\mathrm{r}=0.270 \pm 0.084, \mathrm{p}=0.004)$, while significantly and negatively correlated with CRP (0.302 \pm $0.126 ; 0.026)$. SES was also independently and positively correlated with SEFI at admission $(0.478 \pm 0.166 ; \mathrm{p}=0.009)$ and at the end of hospitalization $(\mathrm{r}=0.875 \pm 0.124 ; \mathrm{p}<0.0001)$.

Seven patients $(7 / 26)$ had no significant progression in handgrip values at the end of hospitalization and 5 had no progression in SES values, whereas 19 patients had a positive progression in handgrip and a positive self-evaluation of muscle strength $\left(\mathrm{chi}^{2}=13.87 ; \mathrm{p}<0.0001\right)$. SES enabled detecting $95 \%$ of patients with progression in muscle strength and detecting $5 / 7$ patients without progression (71\%).

Table 1. Patient characteristics at admission.

\begin{tabular}{ll} 
Sex $(\mathrm{F} / \mathrm{M})$ & $5 / 21(19.2 / 80.8 \%)$ \\
BMI Class & \\
$18.5-24.9$ & $8(30.8 \%)$ \\
$25-29.9$ & $9(34.6 \%)$ \\
$>30$ & $9(34.6 \%)$ \\
IPAQ (before COVID-19) & $9(35.0 \%)$ \\
Low & 9 \\
\hline
\end{tabular}




\begin{tabular}{ll}
\hline Moderate & $13(50.0 \%)$ \\
High & $4(14.4 \%)$ \\
Anosmia/dysgeusia & $8(30.8)$ \\
Dysphagia & $6(23.1 \%)$ \\
Active smoking & $1(3.8 \%)$ \\
ICU before non-ICU & $15(57.7 \%)$ \\
Oxygen therapy & $15(57.7 \%)$ \\
Comorbidities & \\
HBP & $15(57.7 \%)$ \\
Coronary heart disease & $11(42.3 \%)$ \\
Dyslipidemia & $8(30.8 \%)$ \\
Diabetes mellitus & $5(19.2 \%)$ \\
Renal failure & $4(15.4 \%)$ \\
Stroke & $3(11.5 \%)$ \\
Asthma & $2(7.7 \%)$ \\
Apnea & $4(15.4 \%)$ \\
Chronic obstructive bronchitis & $1(3.8 \%)$ \\
Respiratory failure & $1(3.8 \%)$ \\
Cancer & $3(11.5 \%)$ \\
Neurological disease & $2(7.7 \%)$ \\
\hline
\end{tabular}

BMI: body mass index; IPAQ: International Physical Activity Questionnaires; ICU: intensive care unit; HBP: high blood pressure

Table 2. Evolution of nutritional status and muscle strength between admission, discharge and Day-30.

\begin{tabular}{|c|c|c|c|c|c|}
\hline & Before COVID-19 & Admission & Discharge & D30 & p Anova \\
\hline Age (years) & & $68.2 \pm 15.1$ & & & \\
\hline Weight $(\mathrm{Kg})$ & $80.9 \pm 19.7$ & $76.0 \pm 17.3$ & $75.8 \pm 17.6$ & $75.5 \pm 19.8$ & $<0.0001$ \\
\hline BMI & $27.7 \pm 6.4$ & $26.1 \pm 5.5$ & $26.0 \pm 5.6$ & $26.2 \pm 6.1$ & $<0.0001$ \\
\hline Weight variation \% & & $-5.4 \pm 6.3$ & $-0.4 \pm 1.6$ & $1.08 \pm 7.1$ & 0.0016 \\
\hline Total weight variation & & $-5.4 \pm 6.3$ & $-6.1 \pm 4.7$ & $-5.6 \pm 6.8$ & NS \\
\hline Albuminemia $(\mathrm{g} / \mathrm{L})$ & & $30.2 \pm 4.1$ & $30.6 \pm 4.0$ & & NS \\
\hline Transthyretinemia (mg/L) & & $168 \pm 80$ & $186 \pm 84$ & & 0.0220 \\
\hline SEFI & & $5.8 \pm 2.9$ & $7.7 \pm 2.5$ & $9.0 \pm 1.4$ & $<0.0001$ \\
\hline NRS & & $4.7 \pm 1.1$ & & $1.4 \pm 1.3$ & $<0.0001$ \\
\hline NRS $>3$ & & $24 / 26$ & $23 / 26$ & $5 / 22$ & $<0.0001$ \\
\hline Muscle Failure Handgrip $<16 \mathrm{~kg}$ (women) $/ 26 \mathrm{~kg}$ (males) & & 19/26 (73\%) & $11 / 26(42 \%)$ & & 0.0035 \\
\hline Handgrip (mean $(\mathrm{kg}) \pm \mathrm{SD})$ & & $22.8 \pm 13.4$ & $26.5 \pm 14.0$ & & $<0.0001$ \\
\hline SES & & $5.2 \pm 2.4$ & $6.8 \pm 2.0$ & $8.0 \pm 1.5$ & $<0.0001$ \\
\hline SES $<7$ & & $18 / 26(69 \%)$ & $11 / 26(42 \%)$ & $4 / 23(17 \%)$ & 0.001 \\
\hline SARC-F & $1 \pm 1.5$ & $6.5 \pm 2.5$ & & $1.9 \pm 2.6$ & $<0.001$ \\
\hline IPAQ & & & & & 0.024 \\
\hline Low & $9(35.0 \%)$ & & & $12(52 \%)$ & \\
\hline Moderate & $13(50.0 \%)$ & & & $8(35 \%)$ & \\
\hline High & $4(14.4 \%)$ & & & $3(13 \%)$ & \\
\hline $\begin{array}{l}\text { Malnutrition defined by BMI }<18.5 \text { ( } 21 \text { if }>70 \text { years) and/or } \\
\text { weight loss }>5 \% \text { ) }\end{array}$ & & 19/26 (73\%) & $15 / 26(58 \%)$ & 14/23 (61\%) & NS \\
\hline Moderate/severe & & $10 / 9$ & $6 / 9$ & $6 / 8$ & NS \\
\hline $\begin{array}{l}\text { Malnutrition defined by BMI }<18.5 \text { ( } 21 \text { if }>70 \text { years) and/or } \\
\text { weight loss }>5 \% \text { and/or sarcopenia }\end{array}$ & & 21/26 (81\%) & $20 / 26(77 \%)$ & & NS \\
\hline
\end{tabular}

BMI: body mass index; SEFI: Self-Evaluation of Food Intake; NRS: Nutritional Risk Screening; SES: Self Evaluation of Strength; SARC-F: Sarcopenic screening test; IPAQ: International Physical Activity Questionnaires.

\subsection{Nutritional Assessment}

Mean BMI corresponding to fitness weight (stable weight before COVID-19) was $27.7 \pm 6.4 \mathrm{~kg} / \mathrm{m}^{2}$ (mean weight $80.9 \pm$ $19.7 \mathrm{~kg})$, mean admission BMI was $26.1 \pm 5.5$ and mean weight loss was $-5.4 \pm 6.3 \%$ before admission (delay $<1$ month).

At admission, $16 / 26$ patients had a greater than $30 \%$ reduction in food intake (mean $\mathrm{SEFI}=5.8 \pm 2.9$ ), confirmed by evaluation of the return of the meal tray. Patients with anosmia had a significant decrease in food intake comparatively to those without $(5.7 \pm 2.9$ vs. $7.6 \pm 2.5$; $\mathrm{p}=0.037)$. SEFI was negatively and significantly correlated with CRP independently of age $(\mathrm{r}=-0.019 \pm 0.009, \mathrm{p}=0.048)$. One third of the patients $(n=9 ; 35 \%)$ presented severe malnutrition with a weight loss $>10 \%$, or BMI $<17$ or albuminemia $<30 \mathrm{~g} / \mathrm{L}, 4$ of whom had a reduction in food intake greater than $30 \%$. One patient significantly increased his food intake in the first 3 days and 3 underwent enteral tube feeding. Ten patients had moderate malnutrition with a weight loss between $5-10 \%, 8$ of whom had a reduction in food intake greater than $30 \% ; 5$ progressively increased their food intake, 2 patients necessitated enteral tube feeding and 1 patient was considered in palliative situation. Albuminemia 
was below $30 \mathrm{~g} / \mathrm{l}$ for $11 / 26$ patients while $24 / 26$ patients had a $\mathrm{NRS} \geq 3$ (mean=4.7 \pm 1.1 ).

Mean weight loss decreased significantly during hospitalization $(-5.4 \pm 6.3 \%$ before admission and $-0.4 \pm 1.6 \%$ during hospitalization; $\mathrm{p}=0.001)$, while transthyretinemia concomitantly increased significantly $(p=0.022)$ as well as food intake $(\mathrm{p}=0.0001)$.

\subsection{Evolution at 30 Days Post-discharge}

At Day 30, mean SES was $8.0 \pm 1.5$ although 4 of 23 patients had a high suspicion of sarcopenia with a decrease in muscle strength (SES $<7$ (range $3-6$ ).

Globally, IPAQ-SF decreased at D30 $(p=0.024)$. One patient went from high to low activity, one patient decreased from high to moderate activity and 3 patients from moderate to low activity. Sixteen patients did not change their activity ( 8 patients still had a low activity level and 8 still had a moderate activity level), while one patient increased his activity (moderate to high).

Mean weight regain was $1.08 \pm 7.1 \%$ more than at discharge. Total weight loss, in comparison to the weight before Covid-19 was $-5.6 \pm 6.8 \%(\mathrm{p}=\mathrm{NS})$. SEFI increased significantly $(\mathrm{p}<0.0001),($ mean $=9.0 \pm 1.4)$. Five patients had a persistent and significant decrease in food intake (reduced by 30 to $50 \%)$ and a persistent weight loss $(-2.4 \%$ to $-11 \%$ comparatively to before COVID-19).

\section{Discussion}

The present study showed that patients had a good perception of their muscle strength improvement, thus the 10-point verbal or visual analogue scales (SES) could prove useful in assessing the progression of muscle strength. Three quarters of COVID-19 patients admitted in a non-ICU setting had sarcopenia as assessed by hand strength (handgrip evaluation).

One third of these patients had severe malnutrition while one third of patients had moderate malnutrition, despite a majority of overweight or obese patients. Food intake and muscle strength increased during hospitalization except in 7 patients. Despite this nutritional intervention, 30 days after discharge, $1 / 5$ patients exhibited a tenacious muscle failure that was not present prior to COVID-19, and $1 / 5$ had persistent malnutrition. Such patients ultimately require rehospitalization, physiotherapy, dietary intervention, or homecare intervention. Of interest, most of our patients had moderate or high activity level on the IPAQ questionnaire prior to COVID-19, which did not influence muscle strength and its evolution.

While the risk of sarcopenia has been purported to be high on the long-term after severe acute respiratory distress syndrome due to other viruses, such risk has yet to be reported after Covid-19. ICU survivors have been shown to manifest persistent functional limitation 1 year after being discharged from the ICU, largely due to muscle wasting, weakness and fatigue, involving extrapulmonary disease with impaired neuromuscular function as an important determinant of exercise limitation [17]. Protein turnover is also increased in critical illness in the early days of COVID-
19, as a response to massive proteolytic stimuli. Suggested mechanisms include direct muscle invasion by ECoV particles and immune-mediated muscle injury, although satisfactory proof of the direct invasion of SARS CoV into muscle cells is still lacking. In one postmortem skeletal muscle study based on 8 SARS cases, the diagnosis of critical illness myopathy was confirmed based on clinical features Lastly, muscle deconditioning due to immobility with normal finding at electrophysiology stimulation has been associated with diffuse atrophy at muscle biopsy [18].

The present findings strengthen the concept that malnutrition should be systematically and urgently managed in patients affected by COVID-19, while also considering that the immune response has been shown to be weakened by inadequate nutrition [8,9]. Furthermore, overweight and obesity did not protect our patients from malnutrition, an entity that remains to be defined. Fat mass/adipocytes in excess, especially in the form of central obesity, is associated with an inflammatory response that likely contributes to the state of malnutrition. Low-grade chronic systemic inflammation may affect the outcomes of patients with COVID-19 [19].

Specific high-calorie-dense diets in a variety of different textures and consistencies with highly digestible foods and oral nutritional supplements (high protein/high energy) are available for all hospitalized patients, in order to promote the maintenance/increase of protein-calorie intake. However, we are currently recording a significant number of patients reporting severe eating difficulties as a consequence of the symptoms, resulting in the necessity of enteral tube feeding in $1 / 5$ patients. This nutritional intervention permitted to limit weight loss during the hospitalization.

Our study has several limitations, the most noteworthy being its observational nature. This study was furthermore not intended to analyze the effects of management on nutritional effectiveness and survival.

\section{Conclusion}

The present study showed that three quarters of COVID-19 patients admitted in a non-ICU setting presented malnutrition as well as sarcopenia as assessed by hand strength. Screening for malnutrition and muscle failure should be initiated immediately at the onset of care, with the aim of improving nutritional status as well as maintaining muscle mass and physical performance. In clinical practice, during hospitalization, grip strength measured by a handheld dynamometer does not require complex equipment, is inexpensive and easy to administer, even in a COVID unit. On the other hand, the 10-point verbal or visual analogue scales (SES) could prove useful in assessing the long-term progression of muscle strength.

\section{Funding Statement}

No financial and material support. 


\section{Conflict of Interest}

The authors have no conflict of interest to declare.

\section{Statement of Compliance with Standards of Research Involving Humans as Subjects}

All procedures performed in studies involving human participants were in accordance with the ethical standards of the institutional and/or national research committee and with the 1964 Helsinki Declaration and its later amendments or comparable ethical standards.

The study was approved by the Research Commission of the University Hospital of Nancy and the requirement for informed consent was waived by the ethics commission. ClinicalTrials.gov Identifier is NCT04451694.

\section{Statement of Authorship}

D. Quilliot contributed to the conception and design of the research, analysis of the data and drafted the manuscript; $M$ Gerard contributed to the conception and design of the research, acquisition and analysis of the data. PL Nguyen-Thi contributed to the design of the research and analysis of the data; T Bermand contributed to the acquisition of the data; $R$. Jaussaud contributed to the conception and design of the research and acquisition of the data. All authors critically revised the manuscript, agree to be fully accountable for ensuring the integrity and accuracy of the work, and read and approved the final manuscript.

\section{References}

[1] Liu H, Chen S, Liu M, Nie H, Lu H. Comorbid Chronic Diseases are Strongly Correlated with Disease Severity among COVID-19 Patients: A Systematic Review and Meta-Analysis. Aging Dis. 2020; 11: 668-78.

[2] Tong JY, Wong A, Zhu D, Fastenberg JH, Tham T. The Prevalence of Olfactory and Gustatory Dysfunction in COVID-19 Patients: A Systematic Review and Meta-analysis. Otolaryngol Head Neck Surg. 2020; 163: 3-11.

[3] Roos DS, Oranje OJM, Freriksen AFD, Berendse HW, Boesveldt S. Flavor perception and the risk of malnutrition in patients with Parkinson's disease. J Neural Transm (Vienna). 2018; 125: 925-30.

[4] Bruyère $\mathrm{O}$, Beaudart $\mathrm{C}$, Reginster $\mathrm{J}$, Buckinx F, Schoene D, Hirani V, Cooper C, Kanis J, Rizzoli R, McCloskey E, Cederholm T, Cruz-Jentoft A, Freiberger E. Assessment of muscle mass, muscle strength and physical performance in clinical practice: An international survey. Eur Geriatr Med. 2016; 7: 243-246.

[5] Beaudart C, Rolland Y, Cruz-Jentoft AJ, Bauer JM, Sieber C, Cooper C, et al. Assessment of Muscle Function and Physical
Performance in Daily Clinical Practice: A position paper endorsed by the European Society for Clinical and Economic Aspects of Osteoporosis, Osteoarthritis and Musculoskeletal Diseases (ESCEO). Calcif Tissue Int. 2019; 105: 1-14.

[6] Cederholm T, Jensen GL, Correia M, Gonzalez MC, Fukushima R, Higashiguchi $\mathrm{T}$, et al. GLIM criteria for the diagnosis of malnutrition - A consensus report from the global clinical nutrition community. Clin Nutr. 2019; 38: 1-9.

[7] Woo J, Leung J, Morley JE. Validating the SARC-F: a suitable community screening tool for sarcopenia? J Am Med Dir Assoc. 2014; 15: 630-4.

[8] Bouette G, Esvan M, Apel K, Thibault R. A visual analogue scale for food intake as a screening test for malnutrition in the primary care setting: Prospective non-interventional study. Clin Nutr. 2020.

[9] Thibault R, Goujon N, Le Gallic E, Clairand R, Sebille V, Vibert J, et al. Use of 10-point analogue scales to estimate dietary intake: a prospective study in patients nutritionally atrisk. Clin Nutr. 2009; 28: 134-40.

[10] Criteria N-. https://www.mdcalc.com/nutrition-risk-screening2002-nrs-2002.

[11] Thibault R, Quilliot D, Seguin P, Tamion F, Schneider S, Déchelotte P. Nutritional care at hospital during the Covid-19 viral epidemic: Expert opinion from the French-speaking Society for Clinical Nutrition and Metabolism (SFNCM). Nut Clin et Métab. 2020; 34: 97-104.

[12] https://www.uhs.nhs.uk/Media/Southampton-ClinicalResearch/Procedures/BRCProcedures/Procedure-formeasuring-gripstrength-using-the-JAMAR-dynamometer.pdf.

[13] Cruz-Jentoft AJ, Bahat G, Bauer J, Boirie Y, Bruyere O, Cederholm T, et al. Sarcopenia: revised European consensus on definition and diagnosis. Age Ageing. 2019; 48: 16-31.

[14] Krznaric Z, Bender DV, Laviano A, Cuerda C, Landi F, Monteiro R, et al. A simple remote nutritional screening tool and practical guidance for nutritional care in primary practice during the COVID-19 pandemic. Clin Nutr. 2020; 39: 1983-7.

[15] Craig CL, Marshall AL, Sjostrom M, Bauman AE, Booth ML, Ainsworth BE, et al. International physical activity questionnaire: 12-country reliability and validity. Med Sci Sports Exerc. 2003; 35: 1381-95.

[16] Barazzoni R, Bischoff SC, Breda J, Wickramasinghe K, Krznaric Z, Nitzan D, et al. ESPEN expert statements and practical guidance for nutritional management of individuals with SARS-CoV-2 infection. Clin Nutr. 2020; 39: 1631-8.

[17] Herridge MS, Chu LM, Matte A, Tomlinson G, Chan L, Thomas C, et al. The RECOVER Program: Disability Risk Groups and 1-Year Outcome after 7 or More Days of Mechanical Ventilation. Am J Respir Crit Care Med. 2016; 194: 831-44.

[18] Msigwa SS, Wang Y, Li Y, Cheng X. The neurological insights of the emerging coronaviruses. J Clin Neurosci. 2020; 78: 1-7.

[19] Chiappetta S, Sharma AM, Bottino V, Stier C. COVID-19 and the role of chronic inflammation in patients with obesity. Int $\mathrm{J}$ Obes (Lond). 2020; 44: 1790-2. 